\title{
Scrub Typhus: A Neglected Tropical Disease and A Potential Threat in North India
}

\author{
Shadma Yaqoob ${ }^{1}$, Areena Hoda Siddiqui' ${ }^{2 *}$ (D) and Priyanka Shukla ${ }^{1}$ \\ ${ }^{1}$ Department of Microbiology, Era's Medical College and Hospital, Lucknow - 226 003, Uttar Pradesh, India. \\ ${ }^{2}$ Department of Lab Medicine, Sahara Hospital, Lucknow - 226 010, Uttar Pradesh, India.
}

\begin{abstract}
Scrub typhus is a rickettsial disease. It presents clinically with non-specific febrile signs and symptoms and is often under diagnosed. It is a major public health threat. Due to non- specificity of presentation and lack of awareness, there is delay in treatment. The changing epidemiology of scrub typhus should be kept in mind while attending patients with acute febrile illness. This study was undertaken to find the prevalence rate of scrub typhus at our centre and presenting features among cases. This study was done prospectively for a period of six month (July 2017- December 2017). All the patients presenting with fever, chills, headache, myalgia , breathlessness, encephalitis of more than $\mathbf{5}$ days duration were included in the study. Detection was done using rapid immune-chromatography (Scrub Typhus Rapid Kit, SD). All positive samples were reconfirmed by the Scrub Typhus Detect ELISA system for IgG/ Ig Mantibodies (In Bios International Inc., Seattle, WA, USA). As per inclusion criteria a total of 134 samples were received in laboratory. Of 134 samples 38 (28\%) were positive for Scrub Typhus. Most of the cases were male and belonged to age group 31-60yrs age. Co infection was also seen in $18 \%$ of cases. A high index of suspicion should be made, and an early diagnosis and treatment is warranted to prevent disease complications. If left untreated, it can prove fatal. Multi-organ dysfunction especially respiratory impediment and shock are the important dangerous complications that lead to higher case fatality rates.
\end{abstract}

Keywords: Rickettsial disease, scrub typhus, Orientia tsutsugamushi

\footnotetext{
*Correspondence: drareenahoda@rediffmail.com; +919936914436
}

(Received: March 07, 2020; accepted: June 23, 2020)

Citation: Yaqoob S, Siddiqui AH, Shukla P. Scrub Typhus: A Neglected Tropical Disease and A Potential Threat in North India. J Pure App/ Microbiol. 2020;14(2):1589-1593. doi: 10.22207/JPAM.14.2.57

(c) The Author(s) 2020. Open Access. This article is distributed under the terms of the Creative Commons Attribution 4.0 International License which permits unrestricted use, sharing, distribution, and reproduction in any medium, provided you give appropriate credit to the original author(s) and the source, provide a link to the Creative Commons license, and indicate if changes were made. 


\section{INTRODUCTION}

Scrub Typhus is an infectious disease caused by Orientiatsutsugamushi, presenting with acute febrile illness ${ }^{1}$. It is a mite borne Rickettsial disease. It usually occurs after exposure to areas with secondary (scrub) vegetation. It mostly occurs at the end of monsoon season as this season favours the growth mite ${ }^{2,3}$. Early clinical symptoms are characterized by fever, chill, headache, and myalgia. It becomes very difficult for treating physician to differentiate it from other diseases like typhoid, malaria, dengue and leptospirosis. Escharis the diagnostic feature of the disease but it is seen in very few. It is one of the most emerging infections in India and the number of patients is increasing in various hospitals in India. If not diagnosed and treated early; it leads to complications that include acute renal failure, meningoencephalitis, gastrointestinal bleeding, and finally multiple organ failures. The incidence of other infection like leptospirosis, Dengue, Malaria also increases after the rainy season and often present as an acute febrile illness and very difficult to differentiate clinically. Co-infections of these diseases with Scrub Typhus, are rare but have been reported in many studies ${ }^{4,5}$.

\section{MATERIAL AND METHODS}

This study was done to look for Scrub typhus amongst the patients who were admitted in tertiary care hospital with history off ever, chills, headache, and myalgia of more than 5 days duration from July 2017 to December 2017. Outpatient Department (OPD) cases with the same clinical features were also included in the study. One hundred thirty four serum samples were collected and investigated for Dengue (PanBio Dengue Elisa Abbot), typhoid(Typhidot, Reszon Diagnostic, Microgene), Leptospirosis(Panbio Leptospira Elisa, Abbot), Malaria (Binax Now Malaria, Abbot) and Hepatitis ( Liver function test) to rule out these differential diagnosis and also to see, if any associated co-infections are present. The age of the patients ranged from 2 to 90 years. Age wise groups were made that included 3 age groups ie: 0-30yrs, 31-60yrs, and 61-90yrs. The other test like blood culture, urine culture, hepatitis markers were also done in some of the cases of scrub typhus. At the same time samples were used to detect the presence of rickettsial antibodies against O. tsusugamushi by rapid immunochromatography for IgM/IgG(Biolinetsutsugamushi, SD) afterruling out other possible causes. All positive samples were reconfirmed by the Scrub Typhus Detect ELISA system for IgG/IgM Antibodiesas per manufacturers instruction (InBios International Inc., Seattle, WA, USA).

\section{RESULTS}

A total of 134 serum samples were collected from IPD and OPD febrile patients, and then investigated for Typhoid, Dengue, Malaria, Hepatitis, Leptospirosis and Scrub Typhus. The test for Scrub Typhus was done to detect the presence of Rickettsial antibodies by rapid immunochromatography assay and confirmed by the Scrub Typhus Detect ELISA system for IgG/IGM Antibodies. All the positive samples correlated

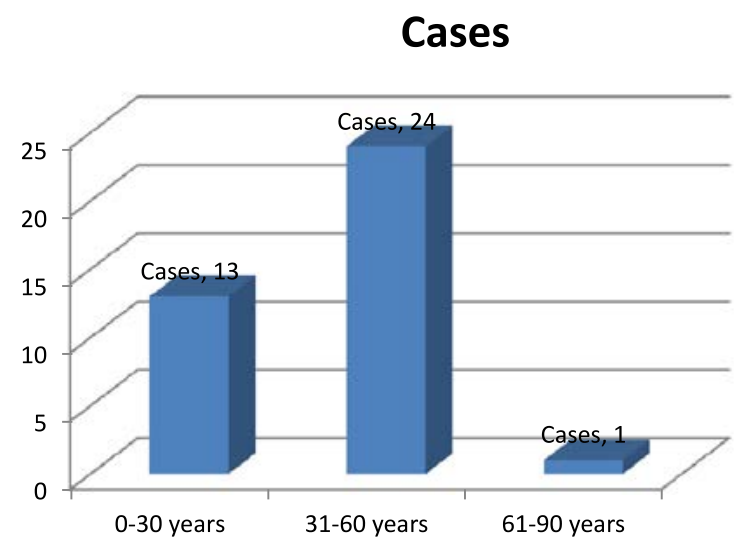

Fig. 1. Age wise prevalence of scrub typhus cases 


\section{Cases}

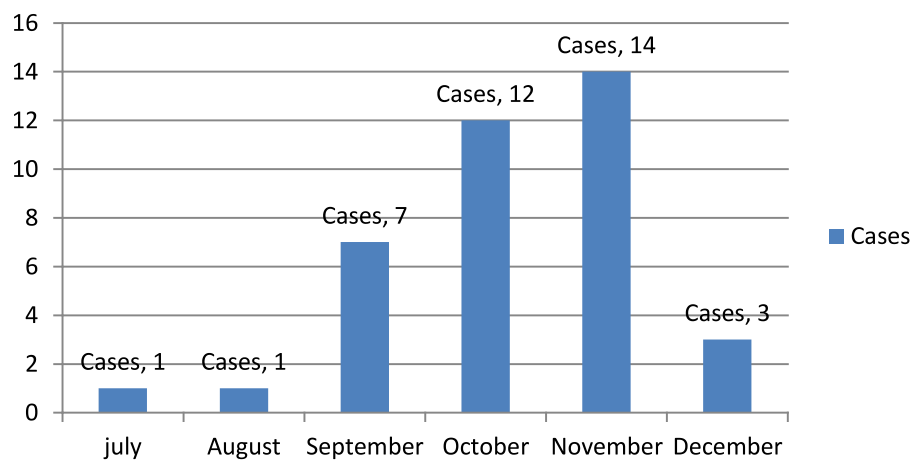

Fig. 2. Month wise distribution of scrub typhus cases

with ELISA result. Of 134 samples 38(\%) were positive for Scrub Typhus, 2 cases having malaria, 4 for Typhoid, 17 were Dengue test positive, 3 for Leptospirosis. Rest of the febrile Patients were treated asymptomatically for common cold, Respiratory infections etc. Agewise groups were made for Scrub Typhus patients that includes 3 age groups ie: 0-30yrs, 31-60yrs, 61-90 yrs as shown in Fig. 1. Maximum cases ie: 24 patients were from 31-60yrs age groups. Males were found to be more infected (63\%) than females (37\%). Most of the cases were reported during winter season. Among 38 positive cases, maximum cases were reported in October and November as shown in Fig. 2. This was just the end of rainy season and suitable for mites. Chances of co-infection of Scrub Typhus with Dengue and Leptospirosis were noted in this study. All 3 were Zoonotic and systemic febrile illnesses with similar signs and symptoms. Usually Dengue cases are maximum during and after monsoon season. In this study also we noted 5 cases of Dengue and Scrub Typhus co-infection may be because of same seasonal increase in Dengue cases also. Scrub Typhus and Leptospirosis Coinfection were also found in 2 cases so $18 \%$ of cases showing co-infections, among which 4 were males and 3 females. Among 7 co infected individuals 4 patients died.

\section{DISCUSSION}

The prevalence of Scrub Typhus have been reported from different part of India by Mittal et al., Gurung et al., Nitin et al. and others ${ }^{6,3,5}$. Presence of Scrub Typhus in Uttar Pradesh were also reported in few other studies ${ }^{7,8}$. The prevalence of Scrub Typhus in our study is $28 \%$.A study from Rajasthan reported $23 \%$ and Sinha et al. reported $25 \%{ }^{9,10}$. Few studies by Vivekanandan M, Gurung and Sharma et al. also found $\geq 30 \%$ of prevalence rate ${ }^{2,3,11}$.

The test for Scrub Typhus was done by rapid immunochromatography assay and all 38 positive cases were also confirmed positive by the Scrub Typhus Detect ELISA system for IgG/IGM Antibodies ${ }^{12}$.

Out of 38 positive cases $26(68.5 \%)$ were reported in October and November. Studies by Vivekanandam et al. and Mahajan et al. ${ }^{2,13}$ reported the majority of cases during and at the end of rainy season which are the active periods of mites.

Three age wise groups were made for Scrub Typhus patients. Among these 31-60yrs age group having highest positivity. Males were found to be more infected than females. Similar findings with higher rate of infections in males $(77 \%)$ was reported by K.S. Roopa et al. and Rajoor et al. ${ }^{14,15}$. We made gender wise distribution of Scrub Typhus infection that showed the higher rate of infection in males compared to females as compared to other studies.

Few patients of Scrub Typhus having coinfection with Dengue, Leptospirosis, Malaria, Typhoid was also reported. It was very difficult to differentiate clinically scrub typhus, leptospirosis and Dengue because of similar sign and symptoms ${ }^{16}$. So we investigated the patients for all these diseases and found 2 cases of Scrub Typhus and Leptospirosis coinfection, 5 cases of 
Dengue coinfection. We found $6 \%$ of Leptospirosis co-infection and $14.7 \%$ of Dengue co-infection Similar studies showing Leptospirosisco-infection and Dengueco-infection were done by Nitin et al., SK Mahajan et al., Sohaib et al. 5,13,16

In our study, 3 females and 1 male died after prolonged hospital stay so the case fatality rate was $10.5 \%$. Nearly similar fatality rate of $13.6 \%$ was reported by Sharmaet al. ${ }^{17}$.

All the positive cases were given doxycycline as drug for treatment and were cured.

\section{CONCLUSION}

It is concluded that in acute febrile cases scrub typhus should also be considered as one of the important differential diagnosis. It should be investigated by Rapid Immunochromatography assay and confirmed by the Scrub Typhus Detect ELISA system to prevent serious complications. Co-infections with Dengue, Leptospirosis, Malaria, Typhoid should also be ruled out in such patients. Immediate treatmentis needed as delay may leads to higher mortality. Most of the patient responded to Doxcycycline, drug of choice for this disease ${ }^{12}$.

Thus, in north India, an increasing awareness about the disease presentation, clinical features, and laboratory findings will help in reducing the mortality from this infectious disease.

\section{ACKNOWLEDGEMENTS}

We are thankful to all the clinicians who have sent samples during the study period

\section{CONFLICT OF INTEREST}

The authors declare that there is no conflict of interest.

\section{AUTHORS' CONTRIBUTIONS}

All authors listed have made a substantial, direct and intellectual contribution to the work, and approved it for publication.

\section{FUNDING}

None.

\section{ETHICS STATEMENT}

Not applicable.

\section{DATA AVAILABILITY}

All datasets generated or analyzed during this study are included in the manuscript

\section{REFERENCES}

1. Chrispal A, Boorugu H, Gopinath KG, et al. Scrub typhus: an unrecognized threat in South India - clinicalprofile and predictors of mortality. Trop Doct. 2010;40(3):12933. https://doi.org/10.1258/td.2010.090452

2. Vivekanandan M, Mani A, Priya YS, Singh AP, Jayakumar S, Purty S. Outbreak ofscrub typhus in Pondicherry. $J$ Assoc Physicians India. 2010;58:24-28.

3. Gurung S, Pradhan J, Bhutia PY. Outbreak of scrub typhus in the North East Himalayan region-Sikkim: an emerging threat. Indian J Med Microbiol. 2013;31: 72-74. https://doi.org/10.4103/0255-0857.108729

4. Chaudhry R, Das A, Premlatha MM, et al. Serological and molecular approaches for diagnosis of leptospirosis in a tertiary care hospital in north India: a 10-year study. Indian J Med Res. 2013;137:785-790.

5. Nitin Gupta et al., Scrub Typhus and Leptospirosis: The fallacy of Diagnosing with IgM Enzyme Linked Immunosorbant Assay. J Microb Biochem Technol. 2016;8:2.

6. Mittal $\mathrm{V}$ et al. Serological evidence of rickettsial infections in Delhi. Indian J Med Res. 2012;135:538541. https://doi.org/10.4103/0971-5916.93416

7. Chandra Dev Pati Tripathi, Mastan Singh, Jyotsna Agarwal, Raj Kumar Kalyan, Chandra Kanta and Virendra Atam. Scrub Typhus in Uttar Pradesh, India-A Prospective Study. Int J Curr Microbiol App Sci. 2017;6(4):977-986. https://doi.org/10.20546/ ijcmas.2017.604.123

8. Rizvi M, Sultan A, Chowdhry M, et al. Prevalence of scrub typhus in pyrexia of unknown origin and assessment of interleukin-8, tumor necrosis factor-alpha, and interferon-gamma levels in scrub typhus-positive patients.Indian J Pathol Microbiol. 2018;61(1):76-80. https://doi.org/10.4103/IJPM. IJPM_644_16

9. Rajendra Prasad Takhar, Motilal Bunkar, Savita Arya, Nitin Mirdha, Arif Mohd. Scrub typhus: A prospective, observational study during an outbreak in Rajasthan, India.

10. Sinha P, Gupta S, Dawra R, Rijhawan P. Recent outbreak of scrub typhus in northwestern part of India. Indian J Med Microbiol. 2014;32:247-250. ESCHCAR. https:// doi.org/10.4103/0255-0857.136552

11. Sharma A, Mahajan S, Gupta ML, Kanga A, Sharma V. Investigation of an outbreak of scrub typhus in the himalayan region of India. Jpn J Infect Dis. 2005;58:208-210.

12. Girija S, Rajan A, Sathiyanarayanan J, Mangaiyarkarasi T, Saban P, Sunil S, Gopal R. Scrub typhus- An emerging disease in South India. IJRRMS. 2013;3(4)

13. Mahajan SK, Rolain JM, Kashyap R, et al. Scrub typhus in Himalayas. Emerg Infect Dis. 2006;12:1590-1592. https://doi.org/10.3201/eid1210.051697 
14. KS Roopa et al. Serodiagnosis of Scrub Typhus at a Tertiary Care Hospital from Southern India. JCDR. 2015;9(11):DC05-DC07. https://doi.org/10.7860/ JCDR/2015/15871.6759

15. Rajoor UG, Gundikeri SK, Sindhur JC, Dhananjaya M. Scrub typhus in adults in ateaching hospital in north Karnataka, 2011-2012. Ann Trop Med Public Health. 2013;6:614-17. https://doi.org/10.4103/17556783.140221
16. Sohaib Ahmad,Minakshi Dhar, Nowneet Kumar Bhat, Nadia Shirazi, Garima Mittal. Rare Co-infection of Malaria, Scrub Typhus and Dengue virus in an Immunocompetent Patient. Int J Curr Microbiol App Sci. 2015;4(5):295-297

17. Navneet Sharma, Manisha Biswal, Abhay Kumar, Kamran Zaman, Sanjay Jain, Ashish Bhalla. Scrub Typhus in a Tertiary Care Hospital in North India. AmJ Trop Med. 2016;95(2)447-451. https://doi. org/10.4269/ajtmh.16-0086 